\title{
ON THE RANGE OF HARMONIC MAPS IN THE PLANE
}

\author{
José G. Llorente \\ Universitat Autònoma de Barcelona, Departament de Matemàtiques \\ 08193 Bellaterra, Barcelona, Spain; jgllorente@mat.uab.cat
}

\begin{abstract}
We give geometrical conditions on the range of a harmonic map in the plane implying that it is constant. This approach is motivated and generalizes Lewis' proof of the classical Little Picard Theorem. We also provide results extending the harmonic Liouville Theorem and the planar version of a result by Murdoch and Kuran.
\end{abstract}

\section{Introduction and main results}

The Little Theorem of Picard says that if an analytic function defined in the complex plane $\mathbf{C}$ omits two complex values then it is constant. Since Picard's original proof, based on the modular function (the universal covering map of $\mathbf{C} \backslash\{0,1\}$ ), different proofs have been found, using Bloch's or Schottky's theorems, normal families and Montel's theorem, curvature of metrics, the so called Heuristic Bloch Principle and also brownian motion (see $[12,14,15,4,7]$ ). Each new proof has contributed in a significative way to broaden and develop the scope of Geometric Function Theory.

During the $80 \mathrm{~s}$ and beginning of the $90 \mathrm{~s}$, a number of works were devoted to generalize Picard's Theorem to real settings. Rickman [13] obtained the first version of Picard's Theorem for quasiregular maps in higher dimensions. Subsequent work of Eremenko-Sodin [6])and Eremenko-Lewis [5] culminated in Lewis' abridged, purely PDE proof of Rickman's theorem [9]. See [2] for yet another simplification of Rickman's theorem based on potential-theoretic methods.

The general idea behind Lewis approach is roughly the following: if a finite family of functions belonging to a specific class (let us say solutions of a PDE) satisfy certain joint value distribution restrictions, then all the functions in the family are constant. Although Lewis proof is valid for the so called Harnack functions (including in particular harmonic functions), the method gives an interesting new proof of the classical Little Picard Theorem. Indeed, let us assume that $f: \mathbf{C} \rightarrow \mathbf{C} \backslash\{0,1\}$ is analytic. Associated to $f$ there are two natural entire harmonic functions, namely $u=\log |f|$ and $v=\log |f-1|$. It is a simple exercise that if $z \in \mathbf{C} \backslash\{0,1\}$, then

$$
\left|\log ^{+}\right| z\left|-\log ^{+}\right| z-1|| \leq \log 2
$$

and

$$
\max (\log |z|, \log |z-1|) \geq-\log 2 .
$$

Little Picard's Theorem is therefore a consequence of the following result, which is contained in [9] with more generality (see also [11]):

https://doi.org/10.5186/aasfm.2020.4550

2010 Mathematics Subject Classification: Primary 30D20, 30D35, 31A05.

Key words: Picard theorem, Liouville theorem, harmonic map, harmonic function, harmonic polynomial.

Partially supported by Spanish Ministry of Sciences, Innovation and Universities under grant MTM2017-85666-P, by Generalitat de Catalunya under grant 2017 SGR 395 and also by the Basque Government through the BERC 2018-21 program and by Spanish Ministry of Sciences, Innovation and Universities: BCAM Severo Ochoa accreditation SEV-2017-0718. 
Theorem. (Lewis) Let $u, v: \mathbf{C} \rightarrow \mathbf{R}$ be harmonic functions satisfying

$$
\begin{gathered}
\left|u^{+}-v^{+}\right| \leq C, \\
\max (u, v) \geq-C
\end{gathered}
$$

for some constant $C>0$. Then $u$ and $v$ are constant.

The proof of Lewis Theorem relies on two fundamental steps. Assuming that $u$ is nonconstant, the first step consists of choosing a sequence of discs at which $u$ exhibits a substantial but controlled oscillation. This sort of "signed Harnack" lemma is the most crucial and technical part of the proof. Secondly, a rescaling method produces two sequences of harmonic functions in the unit disc capturing the behavior of $u$ and $v$ in the chosen sequence of discs. The hypothesis (1.1) and (1.2) together with well known properties of harmonic functions result finally in a contradiction. See Theorem 1.3.11 in [11] for details.

Given two entire harmonic functions $u, v: \mathbf{C} \rightarrow \mathbf{R}$, we will refer to $f=u+$ $i v: \mathbf{C} \rightarrow \mathbf{C}$ as the harmonic map associated to $u$ and $v$. So, for us, an entire harmonic map will be just a pair of harmonic functions defined in the complex plane; in particular no univalence assumption is assumed whatsoever. If $f=u+i v: \mathbf{C} \rightarrow \mathbf{C}$ is a harmonic map we denote its range by $\mathcal{R}_{f}=f(\mathbf{C})$.

Our main motivation for the results in this paper was to reinterpret Lewis theorem in terms of the range $\mathcal{R}_{f}$. As a first basic example in this direction, the harmonic Liouville Theorem ([11]) can be rephrased as follows: if $f=u+i v: \mathbf{C} \rightarrow \mathbf{C}$ is a harmonic map such that $\mathcal{R}_{f}$ is contained in a half-plane, then there exist $a, b, c \in \mathbf{R}$ such that $a u+b v=c$. In particular $\mathcal{R}_{f}$ is a point or a line.

As for Lewis Theorem, it can be read as follows: if $f=u+i v: \mathbf{C} \rightarrow \mathbf{C}$ is a harmonic map and

$$
\mathcal{R}_{f} \subset\left\{u+i v:\left|u^{+}-v^{+}\right| \leq C, \max (u, v) \geq-C\right\} \equiv \mathcal{R}_{L e}
$$

for some constant $C>0$, then $f$ is constant. Observe that the set $\mathcal{R}_{L e}$ in (1.3) is a cross-like neigbourhood of the half-lines $\{u=v \geq 0\},\{u=0, v \leq 0\}$ and $\{v=0, u \leq 0\}$ (see Figure 1.1).

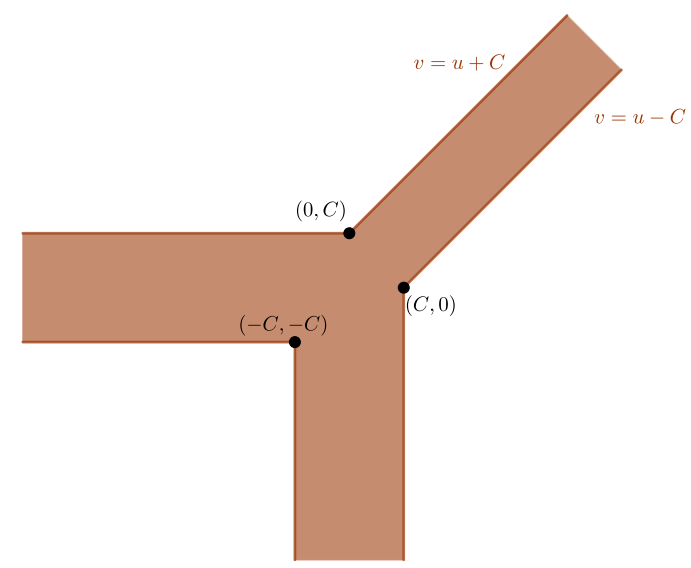

Figure 1.1. The region $\mathcal{R}_{L e}$.

Before stating our main results we need some definitions and notation. Hereafter $\mathbf{D}=\{z \in \mathbf{C}:|z|<1\}$ will denote the unit disc in the complex plane and $\partial \mathbf{D}=$ $\left\{e^{i \theta}: \theta \in \mathbf{R}\right\}$ the unit circle. If $z_{0} \in \mathbf{C}$ and $r>0, D\left(z_{0}, r\right)$ denotes the (open) disc centered at $z_{0}$ of radius $r$. 
Definition 1.1. (Asymptotic directions) Let $\mathcal{R} \subset \mathbf{C}$. We say that $e^{i \theta} \in \partial \mathbf{D}$ is an asymptotic direction of $\mathcal{R}$ if there exist a sequence of points $\left\{w_{n}\right\} \subset \mathcal{R}$ and a sequence of positive numbers $\left\{\varepsilon_{n}\right\}$ with $\varepsilon_{n} \rightarrow 0$ as $n \rightarrow \infty$ such that

$$
\lim _{n \rightarrow \infty} \varepsilon_{n} w_{n}=e^{i \theta}
$$

The set of asymptotic directions of $\mathcal{R}$ will be denoted by $\mathcal{D}(\mathcal{R})$.

Definition 1.1 is strongly motivated by the rescaling method which will be extensively used in section 4 . Note also that our concept of asymptotic direction is broader than the standard one: if, for instance, $\mathcal{R}=\left\{u+i v \in \mathbf{C}: u \geq v^{2}\right\}$, then the positive $u$-semiaxis is the only asymptotic direction, that is, $\mathcal{D}(\mathcal{R})=\{1\}$. Below we include some examples clarifying Definition 1.1 in some specific situations. We refer to section 2 for further properties of asymptotic directions.

Example 1.1. If $\mathcal{R}_{L e}$ is the "Lewis" range set given by (1.3), then $\mathcal{D}\left(\mathcal{R}_{L e}\right)=$ $\left\{e^{i \pi / 4},-1,-i\right\}$.

Example 1.2. If $f=u+i v$, where $u \equiv C$ for some constant $C$ and $v$ is not constant, then, by the harmonic Liouville Theorem, $\mathcal{R}_{f}=\{C+i \mathbf{R}\}$ and $\mathcal{D}\left(\mathcal{R}_{f}\right)=$ $\{i,-i\}$. Analogously, if $v$ is constant and $u$ is not constant then $\mathcal{D}\left(\mathcal{R}_{f}\right)=\{-1,1\}$. If $\mathcal{R}_{f}$ is an arbitrary line in the complex plane, then $\mathcal{D}\left(\mathcal{R}_{f}\right)$ would of course reduce to the two (opposite) directions on $\partial \mathbf{D}$ corresponding to that line.

Example 1.3. Let $f=u+i v$, where $u=x, v=e^{x} \sin y$. Then $\mathcal{R}_{f}=\{u+$ $\left.i v:|v| \leq e^{u}\right\}$ and $\mathcal{D}\left(\mathcal{R}_{f}\right)=\{-1\} \cup\left\{e^{i \theta}:-\pi / 2 \leq \theta \leq \pi / 2\right\}$.

Example 1.4. Let $f=u+i v$, where $u=e^{x} \sin y$ and $v=e^{-x} \sin y$. Then $\mathcal{R}_{f}=\{u+i v: 0<|u v| \leq 1\} \cup\{0\}$ and $\mathcal{D}\left(\mathcal{R}_{f}\right)=\{-1,-i, 1, i\}$.

Example 1.5. Let $0 \leq \alpha<1, a \geq 0, b \in \mathbf{R}$ and $\mathcal{R}=\left\{u+i v: v \leq a|u|^{\alpha}+b\right\}$. Then

$$
\mathcal{D}(\mathcal{R})=\left\{e^{i \theta}:-\pi \leq \theta \leq 0 .\right\}
$$

We describe now the main results of the paper. Recall that two diametrically opposite points on $\partial \mathbf{D}$ are called antipodal. A closer look at the previous examples shows that, with the exception of the Lewis range set $\mathcal{R}_{L e}$ (Example 1.1), the sets of asymptotic directions $\mathcal{D}\left(\mathcal{R}_{f}\right)$ in the rest of examples contain pairs of antipodal points. Our first result says that this must be actually the case.

Theorem 1.1. Let $f=u+i v: \mathbf{C} \rightarrow \mathbf{C}$ be a harmonic map such that $\mathcal{D}\left(\mathcal{R}_{f}\right)$ contains no pair of antipodal points. Then $f$ is constant.

The next two theorems go in a slightly different direction: we discuss assumptions on the range of a harmonic map $f=u+i v$ under which $u$ and $v$ are lineally dependent. Our next result and the corollary below extend the harmonic Liouville Theorem.

Theorem 1.2. Let $f=u+i v: \mathbf{C} \rightarrow \mathbf{C}$ be a harmonic map such that

$$
\mathcal{D}\left(\mathcal{R}_{f}\right) \subset\left\{e^{i \theta}: \alpha-\pi / 2 \leq \theta \leq \alpha+\pi / 2\right\}
$$

for some $\alpha \in \mathbf{R}$. Then there exists $c \in \mathbf{R}$ such that $(\cos \alpha) u+(\sin \alpha) v=c$. In particular $\mathcal{R}_{f}$ is a line or a point.

The next corollary is a consequence of Theorem 1.2 and Example 1.5. 
Corollary 1.1. Let $f=u+i v: \mathbf{C} \rightarrow \mathbf{C}$ be a harmonic map. Suppose that there exist $0 \leq \alpha<1, a \geq 0$ and $b \in \mathbf{R}$ such that

$$
v \leq a|u|^{\alpha}+b
$$

Then $v$ is constant. In particular $\mathcal{R}_{f}$ is a (horizontal) line or a point.

Our third result is motivated by the following classical result of Murdoch [10] and Kuran [8]: if $u$ is a nonconstant harmonic polynomial in $\mathbf{R}^{n}, v$ is harmonic in $\mathbf{R}^{n}$ and $u v \geq 0$ outside a ball, then there is a nonnegative constant $\lambda$ such that $v=\lambda u$. In particular $v$ is also a polynomial. Observe that the functions $u=e^{x} \sin y$, $v=e^{-x} \sin y$ show that the assumption that one of the functions is a polynomial is necessary, even in dimension 2 .

We can restate the Murdoch-Kuran theorem in the plane as follows: if $f=$ $u+i v: \mathbf{C} \rightarrow \mathbf{C}$ is a harmonic map with the additional assumption that $u$ is a nonconstant harmonic polynomial and $f(\mathbf{C} \backslash D(0, R)) \subset\{u+i v: u v \geq 0\}$ for some $R>0$ then there exists $\lambda \geq 0$ such that $v=\lambda u$. In particular $v$ is also a polynomial and $\mathcal{R}_{f}$ is a line. The next result says that, in the plane, the union of the two quadrants $\{u v \geq 0\}$ can be replaced by any cone symmetric respect to the $u$-axis and having the origin as a vertex. Note that, even if the aperture of the cone is exactly $\pi / 2$, this is not, a priori, a direct consequence of the Murdoch-Kuran theorem because a rotation on the $(u, v)$-plane does not preserve the fact that one of the functions is a polynomial. However, we will see that the proof is eventually reduced to the case in which both $u$ and $v$ are polynomials.

Theorem 1.3. Let $f=u+i v: \mathbf{C} \rightarrow \mathbf{C}$ be a harmonic map such that $u$ is a nonconstant harmonic polynomial. Suppose that there exist $a>0$ and $R>0$ such that

$$
f(\mathbf{C} \backslash D(0, R)) \subset\{u+i v:|u| \leq a|v|\}
$$

Then there exists $b \in \mathbf{R}$, with $|b| \leq a$ such that $u=b v$. In particular, $v$ is also a polynomial and $\mathcal{R}_{f}$ is a line.

The structure of the paper is as follows. In section 2 we show some basic properties of asymptotic directions. Section 3 reviews Lewis Lemma and a slight generalization that will be needed later. Section 4 discusses the rescaling method. Finally, sections 5, 6 and 7 are devoted to the proofs of Theorem 1.1, 1.2 and 1.3, respectively.

Acknowledgments. Part of this research has been done when the author was visiting the Basque Center for Applied Mathematics (BCAM). He specially thanks Carlos Pérez for his support and the staff and researchers at BCAM for the hospitality and stimulating work atmosphere. The author also thanks the anonymous referees for helpful remarks.

\section{Basic facts about asymptotic directions}

We remind that for $\mathcal{R} \subset \mathbf{C}$, then $\mathcal{D}(\mathcal{R}) \subset \partial \mathbf{D}$ denotes the subset of asymptotic directions associated to $\mathcal{R}$.

Proposition 2.1. Let $\mathcal{R} \subset \mathbf{C}$. Then $e^{i \theta} \in \mathcal{D}(\mathcal{R})$ if and only if there is a sequence $\left\{w_{n}\right\} \subset \mathcal{R}$ such that $\left|w_{n}\right| \rightarrow \infty$ and $\left|w_{n}\right|^{-1} w_{n} \rightarrow e^{i \theta}$ as $n \rightarrow \infty$.

Proof. Let $e^{i \theta} \in \mathcal{D}(\mathcal{R})$. Choose $\left\{w_{n}\right\} \subset \mathcal{R}$ and $\varepsilon_{n} \rightarrow 0$ such that $\varepsilon_{n} w_{n} \rightarrow e^{i \theta}$ as $n \rightarrow \infty$. In particular $\varepsilon_{n}\left|w_{n}\right| \rightarrow 1$ and

$$
\varepsilon_{n} w_{n}-\left|w_{n}\right|^{-1} w_{n}=\left(\varepsilon_{n}\left|w_{n}\right|-1\right)\left|w_{n}\right|^{-1} w_{n} \rightarrow 0 \quad \text { as } \quad n \rightarrow \infty
$$


which shows that $\left|w_{n}\right|^{-1} w_{n} \rightarrow e^{i \theta}$ as $n \rightarrow \infty$. Conversely, if $\left\{w_{n}\right\} \subset \mathcal{R}$ such that $\left|w_{n}\right| \rightarrow \infty$ and $\left|w_{n}\right|^{-1} w_{n} \rightarrow e^{i \theta}$, then take $\varepsilon_{n}=\left|w_{n}\right|^{-1} \rightarrow 0$. Then $\varepsilon_{n} w_{n} \rightarrow e^{i \theta}$ so $e^{i \theta} \in \mathcal{D}(\mathcal{R})$.

Proposition 2.2. Let $\mathcal{R} \subset \mathrm{C}$.

a) If $\mathcal{R}$ is bounded, then $\mathcal{D}(\mathcal{R})=\varnothing$.

b) If $\mathcal{R}$ is unbounded, then $\mathcal{D}(\mathcal{R})$ is a nonempty, closed subset of $\partial \mathbf{D}$.

Proof. Part a) automatically follows from the definition of asymptotic direction. To prove b), choose a sequence $\left\{w_{n}\right\} \subset \mathcal{R}$ such that $\left|w_{n}\right| \rightarrow \infty$ as $n \rightarrow \infty$. After taking a subsequence we may already assume from compactness that

$$
\lim _{n \rightarrow \infty} \frac{w_{n}}{\left|w_{n}\right|}=e^{i \theta}
$$

for some $\theta \in[0,2 \pi)$, which shows that $e^{i \theta} \in \mathcal{D}(\mathcal{R})$.

To see that $\mathcal{D}(\mathcal{R})$ is closed, suppose that $e^{i \theta_{k}} \in \mathcal{D}(\mathcal{R})$ and $e^{i \theta_{k}} \rightarrow e^{i \theta}$ as $k \rightarrow$ $\infty$, where $\theta_{k}, \theta \in[0,2 \pi)$. By Proposition 2.1 we can choose $w_{k} \in \mathcal{R}$ such that $\left.|| w_{k}\right|^{-1} w_{k}-e^{i \theta_{k}} \mid<1 / k$ so $\left|w_{k}\right|^{-1} w_{k} \rightarrow e^{i \theta}$ as $k \rightarrow \infty$. By Proposition $2.1, e^{i \theta} \in \mathcal{D}(\mathcal{R})$. Therefore $\mathcal{D}(\mathcal{R})$ is closed.

Corollary 2.1. If $f=u+i v: \mathbf{C} \rightarrow \mathbf{C}$ is a nonconstant harmonic map and $\mathcal{R}_{f}=f(\mathbf{C})$ then $\mathcal{D}\left(\mathcal{R}_{f}\right)$ is a nonempty, closed subset of $\partial \mathbf{D}$.

Proof. From the harmonic version of Liouville Theorem, $\mathcal{R}_{f}$ is unbounded. Then apply part b) of Proposition 2.2.

The following elementary proposition collects some particular situations.

Proposition 2.3. Let $f=u+i v: \mathbf{C} \rightarrow \mathbf{C}$ be a harmonic map and $\mathcal{R}_{f}=f(\mathbf{C})$.

a) If $\mathcal{R}_{f}$ is bounded, then $f$ is constant and $\mathcal{D}\left(\mathcal{R}_{f}\right)=\varnothing$.

b) If $u$ is constant and $v$ is not constant, then $\mathcal{R}_{f}$ is a vertical line in the $(u, v)$ plane and $\mathcal{D}\left(\mathcal{R}_{f}\right)=\{-i, i\}$.

c) If $v$ is constant and $u$ is not constant, then $\mathcal{R}_{f}$ is a horizontal line in the $(u, v)$-plane and $\mathcal{D}\left(\mathcal{R}_{f}\right)=\{-1,1\}$.

Proof. Part a) is a direct consequence of part a) in Proposition 2.2. To prove b) observe first that, by the harmonic version of Liouville Theorem, $v(\mathbf{C})=\mathbf{R}$ so $\mathcal{R}_{f}$ is a vertical line in the $(u, v)$-plane. It easily follows from the definition of asymptotic direction that $\mathcal{D}\left(\mathcal{R}_{f}\right)=\{-i, i\}$. Part c) is analogous.

Proposition 2.4. Let $\mathcal{R} \subset \mathbf{C}$ be unbounded and let $\mathcal{D}(\mathcal{R}) \subset \partial \mathbf{D}$ be its associated set of asymptotic directions.

a) If $I \subset \partial \mathbf{D}$ is an open arc such that $I \cap \mathcal{D}(\mathcal{R})=\varnothing$, then for any closed arc $J \subset I$ there exists $\rho>0$ such that $\mathcal{R} \cap\left\{r e^{i \theta}: r \geq \rho, e^{i \theta} \in J\right\}=\varnothing$.

b) If $I \subset \partial \mathbf{D}$ is an open arc and $\mathcal{R} \cap\left\{r e^{i \theta}: r \geq \rho, e^{i \theta} \in I\right\}=\varnothing$ for some $\rho>0$, then $I \cap \mathcal{D}(\mathcal{R})=\varnothing$.

Proof. Suppose that a) does not hold. Then there are sequences $r_{n} \rightarrow \infty$ and $e^{i \theta_{n}} \in J$ such that $w_{n}=r_{n} e^{i \theta_{n}} \in \mathcal{R}$. Since $J$ is closed, there is a subsequence $e^{i \theta_{n_{k}}} \rightarrow e^{i \theta}$ as $k \rightarrow \infty$, for some $e^{i \theta} \in J$. Then $\left|w_{n_{k}}\right|^{-1} w_{n_{k}} \rightarrow e^{i \theta}$, so $e^{i \theta} \in \mathcal{D}(\mathcal{R})$ by Proposition 2.1. This contradiction proves a).

To prove b), suppose that $e^{i \theta} \in I \cap \mathcal{D}(\mathcal{R})$. By Proposition 2.1, choose $w_{n}=$ $\left|w_{n}\right| e^{i \theta_{n}} \in \mathcal{R}$ such that $\left|w_{n}\right| \rightarrow \infty$ and $e^{i \theta_{n}} \rightarrow e^{i \theta}$ as $n \rightarrow \infty$. Since $I$ is open and $\left|w_{n}\right| \rightarrow \infty$ then $e^{i \theta_{n}} \in I$ and $\left|w_{n}\right| \geq \rho$ for $n$ large enough, so for such $n$ 's, 
$w_{n} \in \mathcal{R} \cap\left\{r e^{i \theta}: r \geq \rho, e^{i \theta} \in J\right\}$. This contradicts the hypothesis and therefore shows b).

We will need the following elementary lemma.

Lemma 2.1. If $E \subset \partial \mathbf{D}$ is closed and contains no pair of antipodal points, then there is $\xi \in \partial \mathbf{D}$ such that $\{-i \xi, \xi, i \xi\} \subset \partial \mathbf{D} \backslash E$.

Proof. Assume that $E \neq \emptyset$ because otherwise the result is trivial. We claim that it is enough to show that $\partial \mathbf{D} \backslash E$ contains two antipodal points. Indeed, if $\{\zeta,-\zeta\} \subset \partial \mathbf{D} \backslash E$ for some $\zeta \in \partial \mathbf{D}$ then, by hypothesis, either $i \zeta \in \partial \mathbf{D} \backslash E$ or $-i \zeta \in \partial \mathbf{D} \backslash E$ and the lemma follows.

To prove the claim, note that $\partial \mathbf{D} \backslash E$ is a (countable) union of open arcs in $\partial \mathbf{D}$. Let $I$ be one of such arcs and let $z_{0} \in E$ be one of the end-points of $I$. By hypothesis, $-z_{0} \in \partial \mathbf{D} \backslash E$, which is open, so there is an open arc $J$ centered at $-z_{0}$ such that $J \subset \partial \mathbf{D} \backslash E$. The claim follows from the observation that $(-I) \cap J \neq \emptyset$, where $-I=\{-z: z \in I\}$.

For $\xi=e^{i \alpha} \in \partial \mathbf{D}$ and $0<\phi<\pi / 2$ we denote by $\mathcal{C}_{\xi, \phi}$ (resp. $\mathcal{C}_{\xi, \phi}^{+}$) the whole cone (resp. half cone) with vertex at the origin, axis parallel to $\xi$ and aperture $2 \phi$, that is:

$$
\begin{aligned}
& \mathcal{C}_{\xi, \phi}=\left\{t e^{i \theta}: t \in \mathbf{R},|\theta-\alpha| \leq \phi\right\}, \\
& \mathcal{C}_{\xi, \phi}^{+}=\left\{t e^{i \theta}: t \geq 0,|\theta-\alpha| \leq \phi\right\} .
\end{aligned}
$$

Lemma 2.2. Let $f=u+i v: \mathbf{C} \rightarrow \mathbf{C}$ be a harmonic map and $\mathcal{R}_{f}=f(\mathbf{C})$. Suppose that $\mathcal{D}\left(\mathcal{R}_{f}\right)$ contains no pair of antipodal points. Then there are $\xi \in \partial \mathbf{D}$, $0<\phi<\pi / 2$ and $\rho>0$ such that

$$
\mathcal{R}_{f} \subset \bar{D}(0, \rho) \cup\left(\mathbf{C} \backslash\left(\mathcal{C}_{i \xi, \phi} \cup \mathcal{C}_{\xi, \phi}^{+} \cup\right)\right),
$$

that is, outside some disc centered at the origin, $\mathcal{R}_{f}$ does not intersect the union of a whole cone and a half cone having the origin as a vertex and orthogonal axes.

Proof. By Corollary 2.1 and Lemma 2.1, there exists $\xi=e^{i \alpha} \in \partial \mathbf{D}$ such that $\{-i \xi, \xi, i \xi\} \subset \partial \mathbf{D} \backslash \mathcal{D}\left(\mathcal{R}_{f}\right)$. Since $\partial \mathbf{D} \backslash \mathcal{D}\left(\mathcal{R}_{f}\right)$ is open, the conclusion follows from part a) of Proposition 2.4.

Corollary 2.2. Let $g: \mathbf{C} \rightarrow \mathbf{C}$ be a harmonic map such that $\mathcal{D}\left(\mathcal{R}_{g}\right)$ contains no antipodal points. Then there are $\theta \in[0,2 \pi), \rho>0$ and $a>1$ such that if $f=e^{i \theta} g$, then

$$
\mathcal{R}_{f} \subset \bar{D}(0, \rho) \cup\left(\{u+i v:|v| \leq a|u|\} \backslash\left\{u+i v:|v| \leq-\frac{1}{a} u\right\}\right) .
$$

Proof. Let $\xi \in \partial \mathbf{D}$ be associated to $g$ as in Lemma 2.2 and choose $\theta \in[0,2 \pi)$ so that $e^{i \theta} \xi=-1$. The conclusion readily follows from Lemma 2.2.

\section{Lewis Lemma}

Lewis' proof of Picard's theorem is based on a technical lemma which controls the oscillation of a harmonic function near its zeros (see [9], where it was proved for the more general class of Harnack functions). We state here a version for harmonic functions in the plane (see [11], Lemma 1.3.12).

Lemma 3.1. Let $u$ be harmonic in the disc $D(0, R)$ and continuous in the closed disc $\bar{D}(0, R)$ such that $u(0)=0$. Then there exists a disc $D(z, \rho) \subset D(0, R)$ such 
that

$$
\begin{aligned}
u(z) & =0, \\
M(u, 0, R / 2) & \leq C_{0} M(u, z, \rho), \\
M(u, z, \rho) & \leq C_{0} M(u, z, \rho / 2)
\end{aligned}
$$

for some absolute constant $C_{0}>0$.

Corollary 3.1. Let $u$ be a non constant harmonic function in $\mathbf{C}$. Then there exists a sequence of discs $\left\{D\left(z_{n}, \rho_{n}\right)\right\}$ such that, for all $n$,

$$
\begin{aligned}
u\left(z_{n}\right) & =0, \\
\lim _{n \rightarrow \infty} M\left(u, z_{n}, \rho_{n}\right) & =+\infty, \\
M\left(u, z_{n}, \rho_{n}\right) & \leq C M\left(u, z_{n}, \rho_{n} / 2\right)
\end{aligned}
$$

for some absolute constant $C>0$.

Proof. We may assume that $u(0)=0$. Since $u$ is not constant then $M(u, 0, R) \uparrow$ $+\infty$ as $R \uparrow+\infty$. Take a sequence $R_{n} \rightarrow+\infty$ and apply Lemma 3.1 to the discs $D\left(0, R_{n}\right)$. We then get discs $D\left(z_{n}, \rho_{n}\right)$ such that (3.4) and (3.6) hold. Finally, (3.5) also holds, since $M\left(u, 0, R_{n} / 2\right) \leq C M\left(u, z_{n}, \rho_{n}\right)$ by (3.2) and $M\left(u, 0, R_{n} / 2\right) \rightarrow+\infty$ as $n \rightarrow \infty$.

For further applications to harmonic maps we will need yet a refinement of Corollary 3.1. We include an elementary lemma first.

Lemma 3.2. Let $u$ be harmonic in $D\left(z_{0}, r\right)$ and continuous in $\bar{D}\left(z_{0}, r\right)$ such that $u\left(z_{0}\right)=0$. Then

$$
M\left(|u|, z_{0}, \frac{2}{3} r\right) \leq 4 M\left(u, z_{0}, r\right)
$$

Proof. Put $M=M\left(u, z_{0}, r\right)$ and let $v=M-u$. Then $v$ is harmonic and positive in $D\left(z_{0}, r\right)$. Now by Harnack's inequality,

$$
M\left(v, z_{0}, \frac{2}{3} r\right) \leq 5 v\left(z_{0}\right)=5 M
$$

implying that $-4 M \leq u \leq M$ in $D\left(z_{0}, \frac{2}{3} r\right)$, so $M\left(|u|, z_{0}, \frac{2}{3} r\right) \leq 4 M$.

Corollary 3.2. Let $u$ be a nonconstant harmonic function in $\mathbf{C}$. Then there exists a sequence of discs $D\left(z_{n}, r_{n}\right)$ such that

$$
\begin{aligned}
u\left(z_{n}\right) & =0 \\
\lim _{n \rightarrow \infty} M\left(u, z_{n}, r_{n}\right) & =+\infty \\
M\left(|u|, z_{n}, r_{n}\right) & \leq C_{0} M\left(u, z_{n}, \frac{3}{4} r_{n}\right)
\end{aligned}
$$

for some absolute constant $C_{0}>0$.

Proof. Let $\left\{D\left(z_{n}, \rho_{n}\right)\right\}$ be the sequence of discs provided by Corollary 3.1 and set $r_{n}=\frac{2}{3} \rho_{n}$. Then (3.7) is automatic. By (3.6) and Lemma 3.2,

$$
M\left(|u|, z_{n}, r_{n}\right)=M\left(|u|, z_{n}, \frac{2}{3} \rho_{n}\right) \leq 4 M\left(u, z_{n}, \rho_{n}\right) \leq 4 C M\left(u, z_{n}, \frac{3}{4} r_{n}\right)
$$

which proves (3.9). (3.8) is consequence of (3.5), (3.6) and the fact that $M\left(u, z_{n}, r_{n}\right)=$ $M\left(u, z_{n}, \frac{2}{3} \rho_{n}\right) \geq M\left(u, z_{n}, \rho_{n} / 2\right)$. 


\section{The rescaling method and consequences}

Let $f=u+i v: \mathbf{C} \rightarrow \mathbf{C}$ be a harmonic map such that $\mathcal{D}\left(\mathcal{R}_{f}\right)$ contains no pair of antipodal points. After a rotation we may assume, according to Corollary (2.2), that there exist $a>1$ and $\rho>0$ such that

$$
\mathcal{R}_{f} \subset \bar{D}(0, \rho) \cup\left(\{u+i v:|v| \leq a|u|\} \backslash\left\{u+i v:|v| \leq-\frac{1}{a} u\right\}\right)
$$

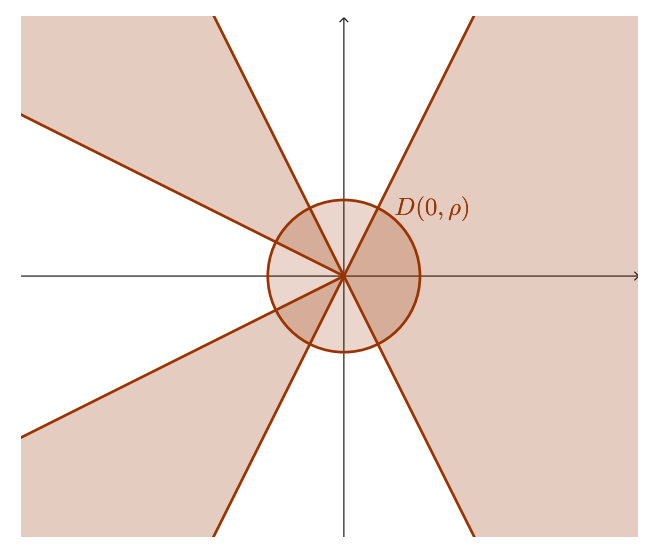

Figure 4.1. The set in the right hand side of (4.1).

Let $\alpha=\arctan (1 / a) \in(0, \pi / 4)$. Then it follows from (4.1) and the definition of asymptotic directions that

$$
\mathcal{D}\left(\mathcal{R}_{f}\right) \subset I_{\alpha}
$$

where

$$
I_{\alpha}=\left\{e^{i \theta}: \theta \in\left[-\frac{\pi}{2}+\alpha, \frac{\pi}{2}-\alpha\right] \cup\left[\frac{\pi}{2}+\alpha, \pi-\alpha\right] \cup\left[\pi+\alpha, \frac{3 \pi}{2}-\alpha\right]\right\}
$$

Assume that $f$ is not constant. Then both $u$ and $v$ must be nonconstant because otherwise $\mathcal{D}\left(\mathcal{R}_{f}\right)$ would contain antipodal points, according to Proposition (2.3). Now, starting from $u$, let $\left\{D\left(z_{n}, r_{n}\right)\right\}$ be a sequence of discs as in Corollary (3.2) and define the following two sequences of harmonic functions in $\mathbf{D}$ :

$$
u_{n}(z)=\frac{u\left(z_{n}+r_{n} z\right)}{M_{n}}, \quad v_{n}(z)=\frac{v\left(z_{n}+r_{n} z\right)}{M_{n}}
$$

where $M_{n}=M\left(|u|, z_{n}, r_{n}\right)$. From Corollary (3.2) it follows that

$$
\begin{aligned}
u_{n}(0) & =0, \\
\left|u_{n}\right| & \leq 1, \\
M\left(u_{n}, 0,3 / 4\right) & \geq C_{0}^{-1}>0 .
\end{aligned}
$$

Also, we get from (4.1) that $\left|v_{n}\right| \leq \max (a, L)$, where

$$
L=\sup _{n} \frac{M(|v|, 0, \rho)}{M_{n}} .
$$

Observe that $L<\infty$, since $M_{n} \rightarrow+\infty$ as $n \rightarrow \infty$. Then both $\left\{u_{n}\right\}$ and $\left\{v_{n}\right\}$ are uniformly bounded sequences of harmonic functions in D. By Harnack's theorem, there exists a subsequence $\left\{n_{k}\right\}$ and harmonic functions $U$ and $V$ in $\mathbf{D}$ such that

$$
u_{n_{k}} \rightarrow U, \quad v_{n_{k}} \rightarrow V
$$


uniformly in compact sets of D. Note that, from (4.4), (4.5) and (4.6) it follows that

$$
\begin{aligned}
U(0) & =0, \\
|U| & \leq 1, \\
M(U, 0,3 / 4) & \geq C_{0}^{-1}>0 .
\end{aligned}
$$

In particular $U$ is nonconstant.

Definition 4.1. If $U$ and $V$ are as above, we will call $F=U+i V: \mathbf{D} \rightarrow \mathbf{C}$ the rescaled harmonic map associated to the original harmonic map $f=u+i v$. We will denote $\mathcal{R}_{F}=F(\mathbf{D})$ the range of $F$.

The following elementary proposition shows how $\mathcal{R}_{F}$ is related to $\mathcal{D}\left(\mathcal{R}_{f}\right)$, the set of asymptotic directions of the original harmonic map $f=u+i v$.

Proposition 4.1. If $F=U+i V$ is as above, then

$$
\mathcal{R}_{F} \subset\left\{r e^{i \theta}: r \geq 0, e^{i \theta} \in \mathcal{D}\left(\mathcal{R}_{f}\right)\right\}
$$

Proof. Let $z \in \mathbf{D}$. By definition of $U$ and $V$ there exists a sequence of complex numbers $\left\{z_{k}\right\} \subset \mathbf{C}$ and two sequences of positive numbers $\left\{r_{k}\right\},\left\{M_{k}\right\}$ with $M_{k} \rightarrow$ $+\infty$ as $k \rightarrow \infty$ such that if $w_{k}=u\left(z_{k}+r_{k} z\right)+i v\left(z_{k}+r_{k} z\right)$, then

$$
\lim _{k \rightarrow \infty} \frac{w_{k}}{M_{k}}=U(z)+i V(z),
$$

so, in particular $\left|w_{k}\right| \rightarrow \infty$ as $k \rightarrow \infty$. If $U(z)+i V(z)=0$, then there is nothing to prove. If $U(z)+i V(z)=R e^{i \beta} \neq 0$, then we get

$$
\lim _{k \rightarrow \infty} \frac{w_{k}}{\left|w_{k}\right|}=e^{i \beta}
$$

Since $w_{k} \in \mathcal{R}_{f}$, the conclusion follows from Proposition (2.1).

According to (4.2), (4.3) we obtain the following consequence.

Corollary 4.1. If $F=U+i V$ is as above, then

$$
\mathcal{R}_{F} \subset\left\{r e^{i \theta}: r \geq 0, e^{i \theta} \in I_{\alpha}\right\},
$$

where $I_{\alpha}$ is as in (4.3). In particular,

$$
\{U=0\} \subset\{V=0\} \subset\{U \geq 0\} .
$$

\section{Proof of Theorem 1.1}

We assume from the beginning that $f=u+i v: \mathbf{C} \rightarrow \mathbf{C}$ is a nonconstant harmonic map (or, equivalently, $\mathcal{D}\left(\mathcal{R}_{f}\right) \neq \varnothing$ by Proposition 2.2). The aim of this section is to derive a contradiction from the further assumption that $\mathcal{D}\left(\mathcal{R}_{f}\right)$ does not contain any pair of antipodal points. Such a contradiction would prove Theorem 1.1. According to section 4 , we may assume that the rescaled harmonic map $F=U+i V: \mathbf{D} \rightarrow \mathbf{C}$ associated to $f$ as in Definition 4.1 satisfies (4.10) and (4.11).

Before starting the proof of Theorem 1.1, some remarks about the local structure of the zero set of a harmonic function in the plane are in order. Suppose that $U$ is a (nonconstant) harmonic function defined in a neighborhood of the origin in the complex plane such that $U(0)=0$. It follows by elementary complex analysis that there exist $r>0$, an integer $n \geq 1$ (the multiplicity of the zero) and a conformal map $\phi$ in $D(0, r)$ such that $U(z)=\mathfrak{R e}(\phi(z))^{n}$ for $z \in D(0, r)$. This shows in particular that the set

$$
\{U=0\} \cap D(0, r)
$$


consists of a union of $n$ analytic curves intersecting at the origin at angle $2 \pi / n$. The complement (in $D(0, r)$ ) of such curves is a "petal-like" region consisting of $2 n$ curvilinear sectors meeting at angle $2 \pi / n$ at the origin such that the sign of $U$ successively alternates in those sectors. The canonical model is, of course, the function $U=\mathfrak{R e}\left(z^{n}\right)$.

The following lemma is a sort of "cleaning" result saying that the inclusion (4.10) can be locally improved.

Lemma 5.1. Let $F=U+i V: \mathbf{D} \rightarrow \mathbf{C}$ be the rescaled harmonic map associated to $f=u+i v$, satisfying (4.10) and (4.11). Then there exists $r>0$ such that

$$
\{U=0\} \cap D(0, r)=\{V=0\} \cap D(0, r) .
$$

Furthermore, either $U V \geq 0$ or $U V \leq 0$ in $D(0, r)$. In particular, either

$$
F(D(0, r)) \subset\left\{r e^{i \theta}: r \geq 0, \theta \in\left[0, \frac{\pi}{2}-\alpha\right] \cup\left[\pi+\alpha, \frac{3 \pi}{2}-\alpha\right]\right\}
$$

or

$$
F(D(0, r)) \subset\left\{r e^{i \theta}: r \geq 0, \theta \in\left[-\frac{\pi}{2}+\alpha, 0\right] \cup\left[\frac{\pi}{2}+\alpha, \pi-\alpha\right]\right\}
$$

for some $0<\alpha<\pi / 4$.

Proof. Recall that $U$ is not constant and that $U(0)=V(0)=0$. Then $V$ cannot be constant, since otherwise $V \equiv 0$, which would imply $U \geq 0$ by (4.11) and therefore $U \equiv 0$ by the Minimum Principle.

Choose $r>0$ so that

$$
\begin{aligned}
& \{U=0\} \cap D(0, r)=\bigcup_{k=1}^{n} \gamma_{k}, \\
& \{V=0\} \cap D(0, r)=\bigcup_{j=1}^{m} \Gamma_{j},
\end{aligned}
$$

where the $\gamma_{k}$ 's and the $\Gamma_{j}$ 's are analytic curves meeting at the origin at angles $2 \pi / n$ and $2 \pi / m$, respectively. Observe that, by (4.11), necessarily $n \leq m$ and each $\gamma_{k}$ is one of the $\Gamma_{j}$ 's. We claim that $n=m$ and that both families of curves actually coincide, so (5.1) follows. The fact that $U V$ has constant sign in $D(0, r)$ would then be a direct consequence of the local structure of the (common) zero set of $U$ and $V$ in $D(0, r)$.

To check the claim, suppose that $n<m$. By the above remarks on the local structure of the zero set, there would be a $j$ such that $\Gamma_{j} \backslash\{0\}$ is contained in one of the curvilinear sectors where $U<0$, which contradicts (4.11). Therefore $n=m$ and we can assume that $\gamma_{k}=\Gamma_{k}$ for $k=1, \cdots, n$.

Remark 5.1. It should be noted that only conditions (4.11) are really used to obtain (5.1) and the fact that $U V$ has constant sign.

Proof of Theorem 1.1. The proof consists of an iterative argument which will result in a contradiction with the fact that $\mathcal{D}\left(\mathcal{R}_{f}\right)$ does not contain pairs of antipodal points. Let $F=U+i V$ and $r_{1}=r$ be as in Lemma 5.1. Assume that $U V \geq 0$ in 
$D\left(0, r_{1}\right)$, so $(5.2)$ holds. Define

$$
\begin{aligned}
& \beta_{1}^{-}=\inf \left\{\theta \in\left(\pi, \frac{3 \pi}{2}\right): \exists R>0 \text { such that } \operatorname{Re}^{i \theta} \in F\left(D\left(0, r_{1}\right)\right)\right\}, \\
& \beta_{1}^{+}=\sup \left\{\theta \in\left(\pi, \frac{3 \pi}{2}\right): \exists R>0 \text { such that } \operatorname{Re}^{i \theta} \in F\left(D\left(0, r_{1}\right)\right)\right\} .
\end{aligned}
$$

Note that $e^{i \beta_{1}^{-}}, e^{i \beta_{1}^{+}} \in \mathcal{D}\left(\mathcal{R}_{f}\right)$ by propositions 4.1 and 2.2. We claim that

$$
\pi<\beta_{1}^{-}<\beta_{1}^{+}<3 \pi / 2 \text {. }
$$

Observe that the first and third inequalities are consequence of (5.2) and that $\beta_{1}^{-} \leq \beta_{1}^{+}$by definition. If $\beta_{1}^{-}=\beta_{1}^{+}=\beta$, then the intersection of $F\left(D\left(0, r_{1}\right)\right)$ with the third quadrant would be contained in a line $V=a U$, where $a=\tan \beta>0$. Then $\{U<0\} \subset\{V-a U=0\}$ in $D\left(0, r_{1}\right)$ and, by unique continuation and the fact that $U$ takes negative values near the origin, we would deduce that $V=a U$ in $\mathbf{D}$ implying that $\mathcal{R}_{F}=F(\mathbf{D})$ is a nontrivial line segment with the origin at its interior. Again by Proposition 4.1 , that would imply that $\mathcal{D}\left(\mathcal{R}_{f}\right)$ contains the antipodal points $\pm e^{i \beta}$, which is a contradiction. This proves (5.4).

Let $a_{1}^{-}=\tan \beta_{1}^{-}$and $a_{1}^{+}=\tan \beta_{1}^{+}$. By the definition of $\beta_{1}^{-}$and $\beta_{1}^{+}$, we also have (again in $\left.D\left(0, r_{1}\right)\right)$ that:

$$
\{U=0\} \subset\left\{V-a_{1}^{ \pm} U=0\right\} \subset\{U \geq 0\},
$$

where the first inclusion in (5.5) is consequence of (5.1) and the second inclusion follows from the fact that if, say, $\left(V-a_{1}^{-} U\right)\left(z_{0}\right)=0$ and $U\left(z_{0}\right)<0$ for some $z_{0} \in D\left(0, r_{1}\right)$ then $V-a_{1}^{-} U \geq 0$ locally around $z_{0}$, by the minimality of $\beta_{1}^{-}$. Again by the Minimum Principle and unique continuation, we would then have $V \equiv a_{1}^{-} U$, implying, as above, that $\mathcal{D}\left(\mathcal{R}_{f}\right)$ would contain the antipodal points $\pm e^{i \beta_{1}^{-}}$, which contradicts the hypothesis. Of course, the same argument works for $V-a_{1}^{+} U$.

It follows from (5.5) and Remark (5.1) that we may perform another "cleaning" argument, now applied to the pairs $U, V-a_{1}^{-} U$ and $U, V-a_{1}^{+} U$. By imitating the proof of Lemma 5.1, it follows that we may choose $0<r_{2} \leq r_{1}$ so that

$$
\{U=0\}=\{V=0\}=\left\{V-a_{1}^{-} U=0\right\}=\left\{V-a_{1}^{+} U=0\right\}
$$

in $D\left(0, r_{2}\right)$. Furthermore, $U\left(V-a_{1}^{-} U\right)$ and $U\left(V-a_{1}^{+} U\right)$ must have constant signs in $D\left(0, r_{2}\right)$, which by inspection turn out to be $U\left(V-a_{1}^{-} U\right) \geq 0$ and $U\left(V-a_{1}^{+} U\right) \leq 0$. In particular,

$$
F\left(D\left(0, r_{2}\right)\right) \subset\left\{U+i V:\left(V-a_{1}^{-} U\right)\left(V-a_{1}^{+} U\right) \leq 0\right\} .
$$

Since $e^{i \beta_{1}^{-}}, e^{i \beta_{1}^{+}} \in \mathcal{D}\left(\mathcal{R}_{f}\right)$ and $\mathcal{D}\left(\mathcal{R}_{f}\right)$ does not contain pairs of antipodal points, it follows that $e^{i\left(\beta_{1}^{-}-\pi\right)}, e^{i\left(\beta_{1}^{+}-\pi\right)} \notin \mathcal{D}\left(\mathcal{R}_{f}\right)$.

Now we are ready to run the next step in the iterative argument. Define

$$
\begin{aligned}
& \beta_{2}^{-}=\sup \left\{\theta \in\left(0, \frac{\pi}{2}\right): \exists R>0 \text { such that } R e^{i \theta} \in F\left(D\left(0, r_{2}\right)\right)\right\}, \\
& \beta_{2}^{+}=\inf \left\{\theta \in\left(0, \frac{\pi}{2}\right): \exists R>0 \text { such that } R e^{i \theta} \in F\left(D\left(0, r_{2}\right)\right)\right\} .
\end{aligned}
$$

Note that, analogously, $e^{i \beta_{2}^{-}}, e^{i \beta_{2}^{+}} \in \mathcal{D}\left(\mathcal{R}_{f}\right)$ and, since $e^{i\left(\beta_{1}^{-}-\pi\right)}$ and $e^{i\left(\beta_{1}^{+}-\pi\right)}$ do not belong to the closed set $\mathcal{D}\left(\mathcal{R}_{f}\right)$, then we get

$$
0<\beta_{1}^{-}-\pi<\beta_{2}^{-}<\beta_{2}^{+}<\beta_{1}^{+}-\pi<\frac{\pi}{2},
$$


where the third inequality in (5.8) follows in the same way than the second inequality in (5.4). By performing another "cleaning" argument similar to the above we may choose $0<r_{3} \leq r_{2}$ so that (5.7) could be improved to

$$
F\left(D\left(0, r_{3}\right)\right) \subset\left\{U+i V:\left(V-a_{2}^{-} U\right)\left(V-a_{2}^{+} U\right) \leq 0\right\},
$$

where $a_{2}^{-}=\tan \beta_{2}^{-}$and $a_{2}^{+}=\tan \beta_{2}^{+}$. In the next step we would obtain $\beta_{3}^{-}$and $\beta_{3}^{+}$ so that

$$
\pi+\beta_{2}^{-}<\beta_{3}^{-}<\beta_{3}^{+}<\pi+\beta_{2}^{+}
$$

and $e^{i \beta_{3}^{-}}, e^{i \beta_{3}^{+}} \in \mathcal{D}\left(\mathcal{R}_{f}\right)$. Continuing this procedure we get sequences $\left\{\beta_{n}^{-}\right\}$and $\left\{\beta_{n}^{+}\right\}$ such that $\left\{\beta_{2 n-1}^{-}\right\}$and $\left\{\beta_{2 n}^{-}\right\}$are increasing, $\left\{\beta_{2 n-1}^{+}\right\}$and $\left\{\beta_{2 n}^{+}\right\}$are decreasing, $e^{i \beta_{n}^{-}}$, $e^{i \beta_{n}^{+}} \in \mathcal{D}\left(\mathcal{R}_{f}\right)$ and

$$
\begin{aligned}
& 0<\beta_{2 n-1}^{-}-\pi<\beta_{2 n}^{-}<\beta_{2 n}^{+}<\beta_{2 n-1}^{+}-\pi<\frac{\pi}{2}, \\
& \pi<\pi+\beta_{2 n}^{-}<\beta_{2 n+1}^{-}<\beta_{2 n+1}^{+}<\pi+\beta_{2 n}^{+}<\frac{3 \pi}{2} .
\end{aligned}
$$

Then it is easy to check that there are $0<\beta^{-} \leq \beta^{+}<\pi / 2$ such that

$$
\lim _{n \rightarrow \infty} \beta_{2 n}^{-}=\beta^{-}, \quad \lim _{n \rightarrow \infty} \beta_{2 n}^{+}=\beta^{+},
$$

and

$$
\lim _{n \rightarrow \infty} \beta_{2 n-1}^{-}=\pi+\beta^{-}, \quad \lim _{n \rightarrow \infty} \beta_{2 n-1}^{+}=\pi+\beta^{+} .
$$

Since $\mathcal{D}\left(\mathcal{R}_{f}\right)$ is closed, this implies in particular that the two antipodal points $e^{i \beta^{-}}$, $e^{i\left(\pi+\beta^{-}\right)}$belong to $\mathcal{D}\left(\mathcal{R}_{f}\right)$, which is the contradiction we were seeking for and finishes the proof of the theorem.

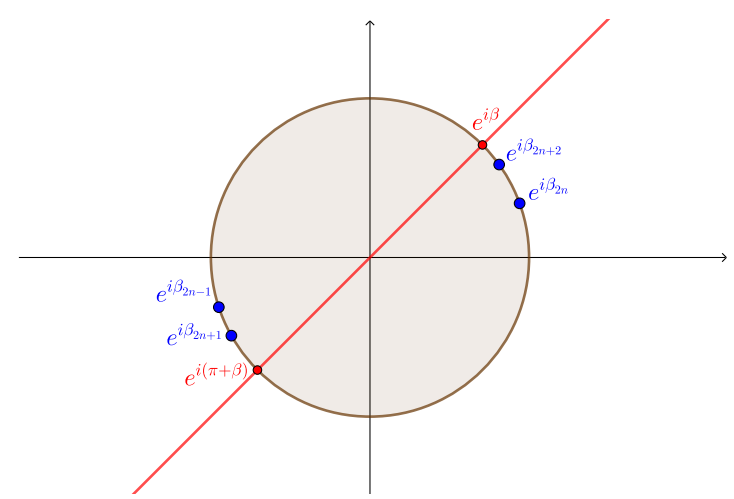

Figure 5.1. Proof of Theorem 1.1.

\section{Proof of Theorem 1.2}

Let $f=u+i v: \mathbf{C} \rightarrow \mathbf{C}$ be a harmonic map and assume, up to a rotation, that

$$
\mathcal{D}\left(\mathcal{R}_{f}\right) \subset\left\{e^{i \theta}:-\pi \leq \theta \leq 0\right\}
$$

If $u$ were constant then $\mathcal{R}_{f}$ would be a vertical line or a point in the $u-v$ plane and, according to Proposition 2.3, either $\mathcal{D}\left(\mathcal{R}_{f}\right)=\varnothing$ or $\mathcal{D}\left(\mathcal{R}_{f}\right)=\{-i, i\}$ so Theorem 1.2 would certainly hold in this case. Assume then that $u$ is not constant. In particular $u$ is unbounded above and below by the harmonic Liouville Theorem and, consequently, $\mathcal{R}_{f}$ cannot be contained in a half-space of the form $\{u \geq c\}$ or $\{u \leq c\}$ for any $c \in \mathbf{R}$.

Now, for each $u \in \mathbf{R}$, let $E_{u}=\left\{v \in \mathbf{R}: u+i v \in \mathcal{R}_{f}\right\}$. By the preceding comments, continuity of $f$ and connectedness of the set $\mathcal{R}_{f}=f(\mathbf{C})$, it follows that $E_{u} \neq \varnothing$ for each $u \in \mathbf{R}$. We claim that $E_{u}$ is bounded above for any $u \in \mathbf{R}$. 
Otherwise, we could find $u \in \mathbf{R}$ and a real sequence $\left\{v_{n}\right\}$ such that $v_{n} \rightarrow+\infty$ as $n \rightarrow+\infty$ and $u+i v_{n} \in \mathcal{R}_{f}$ for all $n$. Then

$$
\lim _{n \rightarrow \infty} \frac{u+i v_{n}}{\left|u+i v_{n}\right|}=i
$$

which, by Proposition 2.1, would imply that $i \in \mathcal{D}\left(\mathcal{R}_{f}\right)$, contradicting (6.1). This proves the claim and allows to define $\Phi: \mathbf{R} \rightarrow[0,+\infty)$ by

$$
\Phi(u)=\max \left\{\sup E_{u}, 0\right\} \text {. }
$$

Lemma 6.1. Let $f=u+i v: \mathbf{C} \rightarrow \mathbf{C}$ be a harmonic map satisfying (6.1) and let $\Phi$ be as in (6.2). Then $\Phi$ is locally bounded and satisfies

$$
\lim _{|u| \rightarrow \infty} \frac{\Phi(u)}{|u|}=0
$$

Proof. Both conclusions are basically consequence of (6.1). If $\Phi$ were not locally bounded, we could find $M>0$ and two real sequences $\left\{u_{n}\right\},\left\{v_{n}\right\}$ such that $\left|u_{n}\right| \leq M$, $u_{n}+i v_{n} \in \mathcal{R}_{f}$ for all $n$ and $v_{n} \rightarrow+\infty$ as $n \rightarrow \infty$. Then

$$
\lim _{n \rightarrow \infty} \frac{u_{n}+i v_{n}}{\left|u_{n}+i v_{n}\right|}=i
$$

so, again by Proposition 2.1, $i \in \mathcal{D}\left(\mathcal{R}_{f}\right)$, contradicting (6.1). The proof of (6.3) follows similar lines: suppose that (6.3) does not hold. Then we can find $\alpha>0$ and a sequence $\left\{u_{n}\right\}$ such that $\left|u_{n}\right| \rightarrow \infty$ and $\Phi\left(u_{n}\right)>\alpha\left|u_{n}\right|$ for all $n$. Assume that $u_{n} \rightarrow+\infty$. Then there exist sequences $\left\{v_{n}\right\}$ and $\left\{\theta_{n}\right\}$ such that $v_{n} \geq \alpha u_{n}$, $\arctan \alpha \leq \theta_{n} \leq \pi / 2, \quad u_{n}+i v_{n} \in \mathcal{R}_{f}$ and

$$
\frac{u_{n}+i v_{n}}{\left|u_{n}+i v_{n}\right|}=e^{i \theta_{n}}
$$

for all $n$. Choose a subsequence $\left\{n_{k}\right\}$ such that $e^{i \theta_{n_{k}}} \rightarrow e^{i \theta}$ as $k \rightarrow \infty$. Then $\arctan \alpha \leq \theta \leq \pi / 2$ and, by Proposition 2.1, $e^{i \theta} \in \mathcal{D}\left(\mathcal{R}_{f}\right)$, which contradicts (6.1). The proof of the lemma is now complete.

Let $f=u+i v: \mathbf{C} \rightarrow \mathbf{C}$ be a harmonic map satisfying (6.1) and let $\Phi$ be as in (6.2). Observe that the definition of $\Phi$ implies that

$$
v(z) \leq \Phi(u(z))
$$

for each $z \in \mathbf{C}$. Then Theorem 1.2 is consequence of the following, even more general result.

Theorem 6.1. Let $w: \mathbf{C} \rightarrow \mathbf{R}$ be subharmonic and $u: \mathbf{C} \rightarrow \mathbf{R}$ be harmonic. Suppose that there exists $\Phi: \mathbf{R} \rightarrow[0,+\infty)$ such that $\Phi$ is locally bounded,

$$
\lim _{|t| \rightarrow \infty} \frac{\Phi(t)}{|t|}=0
$$

and

$$
w(z) \leq \Phi(u(z))
$$

for all $z \in \mathbf{C}$. Then $w$ is constant.

Observe that Theorem 1.2 follows from Theorem 6.1 by choosing $w=v$. For the proof of Theorem 6.1 we will need the following "relative" Maximum Principle (Theorem 3.1.6 in [1]). We recall that $\partial^{\infty} \Omega$ denotes the boundary of $\Omega$ in $\mathbf{C} \cup\{\infty\}$. 
Lemma 6.2. Let $\Omega \subset \mathbf{C}$ be a domain, $s: \Omega \rightarrow \mathbf{R}$ subharmonic and $h: \Omega \rightarrow \mathbf{R}$ harmonic such that $h>0$ in $\Omega$ and

$$
\limsup _{z \rightarrow \xi} \frac{s(z)}{h(z)} \leq 0
$$

for each $\xi \in \partial^{\infty} \Omega$. Then $s \leq 0$ in $\Omega$.

Proof of Theorem 6.1. By the subharmonic Liouville Theorem in the plane (see [11], Chapter 2) it is enough to prove that $w$ is bounded above. If $u$ is constant, then the conclusion follows from (6.4) so we assume hereafter that $u$ is not constant and is therefore unbounded from above and below. We will actually show that if $\Omega$ is any component of $\{u>0\}$ or $\{u<0\}$ then $w \leq \Phi(0)$ in $\Omega$.

Let $\Omega$ be a component of $\{u>0\}$. We pick $z_{0} \in \mathbf{C}$ and $r>0$ such that $\bar{D}\left(z_{0}, r\right) \cap \Omega=\varnothing$ (choose $z_{0}$ and $r$ such that $u<0$ in $\left.\bar{D}\left(z_{0}, r\right)\right)$. Define

$$
s(z)=w(z)-\Phi(0), \quad h(z)=u(z)+\log \left(\frac{\left|z-z_{0}\right|}{r}\right),
$$

and note that $s$ is subharmonic in $\Omega, h$ is harmonic in $\Omega$ and $h>0$ in $\Omega$.

We claim that

$$
\limsup _{z \rightarrow \xi} \frac{s(z)}{h(z)} \leq 0 \quad \text { for all } \quad \xi \in \partial^{\infty} \Omega .
$$

To prove the claim we distinguish the cases i) $\xi \in \partial \Omega \cap \mathbf{C}$ and ii) $\xi=\infty$.

Suppose first that $\xi \in \partial \Omega \cap \mathbf{C}$, in particular $u(\xi)=0$. From subharmonicity and

$$
\limsup _{z \rightarrow \xi} s(z) \leq s(\xi)=w(\xi)-\Phi(0) \leq \Phi(u(\xi))-\Phi(0)=0 .
$$

On the other hand,

$$
\liminf _{z \rightarrow \xi} h(z)=\liminf _{z \rightarrow \xi}\left(u(z)+\log \left(\frac{\left|z-z_{0}\right|}{r}\right)\right)=\log \left(\frac{\left|z-z_{0}\right|}{r}\right)>0,
$$

so case i) follows from (6.8) and (6.9).

For case ii) we need to show that

$$
\limsup _{\substack{z \rightarrow \infty \\ z \in \Omega}} \frac{w(z)-\Phi(0)}{u(z)+\log \left(\frac{\left|z-z_{0}\right|}{r}\right)} \leq 0 .
$$

Fix $\varepsilon>0$. From (6.5) we can choose $t_{0}>0$ such that $\Phi(t)<\varepsilon|t|$ if $|t| \geq t_{0}$. Let

$$
M_{0}=\sup _{\left[0, t_{0}\right]} \Phi
$$

(note that $\Phi$ is locally bounded) and take $|z|$ large enough so that

$$
\left|z-z_{0}\right|>r \exp \left(\frac{M_{0}}{\varepsilon}\right) .
$$

If $u(z)>t_{0}$, then

$$
\frac{\Phi(u(z))-\Phi(0)}{u(z)+\log \left(\frac{\left|z-z_{0}\right|}{r}\right)} \leq \varepsilon .
$$

If $0<u(z) \leq t_{0}$, then from (6.11)

$$
\frac{\Phi(u(z))-\Phi(0)}{u(z)+\log \left(\frac{\left|z-z_{0}\right|}{r}\right)} \leq \frac{M_{0}}{\log \left(\frac{\left|z-z_{0}\right|}{r}\right)}<\varepsilon .
$$


so case ii) also follows. The hypothesis of Lemma 6.2 are then fulfilled and we get $s \leq 0$ in $\Omega$ or, equivalently, $w \leq \Phi(0)$ in $\Omega$. Since $\Omega$ is arbitrary this proves that $w \leq \Phi$ in $\{u>0\}$. An analogous argument, replacing $u$ by $-u$ would show that $w \leq \Phi(0)$ in any component of $\{u<0\}$. Then $w \leq \Phi(0)$ in $\mathbf{C}$ and therefore, $w$ is constant.

\section{Proof of Theorem 1.3}

Note first that $v$ cannot be constant because in that case (1.4) would imply that $u$ is bounded, therefore constant. Suppose that $u$ is a harmonic polynomial of degree $n$. By well known properties of the tracts of harmonic polynomials in the plane (see [3]) we can choose $R^{\prime} \geq R$ such that $\{u \neq 0\} \backslash \bar{D}\left(0, R^{\prime}\right)=\bigcup_{i=1}^{2 n} G_{i}$ where each $G_{i}$ is a "sector-like" connected component, in particular $G_{i} \backslash \bar{D}\left(0, R^{\prime}\right)$ is connected. On the other hand, assumption (1.4) implies

$$
\{u \neq 0\} \backslash \bar{D}\left(0, R^{\prime}\right) \subset\{v \neq 0\} .
$$

We claim that the set $\{v \neq 0\}$ has a finite number of components and therefore $v$ is a harmonic polynomial, by Theorem 1 in [3].

It is well known, from the Maximum Principle, that the components of $\{v \neq 0\}$ are nonempty and unbounded. Let $\Omega$ be one such component and pick $z_{0} \in \Omega$ such that $\left|z_{0}\right|>R^{\prime}$ and $u\left(z_{0}\right) \neq 0$. Then there is a unique $i \in\{1, \cdots, 2 n\}$ such that $z_{0} \in G_{i}$ and the connectedness of $G_{i} \backslash \bar{D}\left(0, R^{\prime}\right)$ implies that $G_{i} \backslash \bar{D}\left(0, R^{\prime}\right) \subset \Omega$. The correspondence $\Omega \rightarrow G_{i}$ is $1-1$, since if two different components $\Omega_{1}$ and $\Omega_{2}$ were assigned to the same $G_{i}$, this would imply that $\emptyset \neq G_{i} \backslash \bar{D}\left(0, R^{\prime}\right) \subset \Omega_{1} \cap \Omega_{2}=\emptyset$ which is a contradiction. This shows that $\{v \neq 0\}$ has, at most, $2 n$ components and therefore $v$ is also a harmonic polynomial.

Consider now the harmonic polynomials $u-a v$ and $u+a v$ and observe that $(a v-u)(a v+u) \geq 0$ outside a disc. By the Murdoch-Kuran theorem (or perhaps a more elementary argument) we deduce that $a v+u=\lambda(a v-u)$ for some $\lambda \geq 0$, which implies that $u=b v$ for some $b \in \mathbf{R}$ with $|b| \leq a$, as desired.

\section{References}

[1] Armitage, D. H., and S. J. Gardiner: Classical potential theory. - Springer-Verlag, 2001.

[2] Bonk, M., and P. Poggi-Corradini: The Rickman-Picard theorem. - Ann. Acad. Sci. Fenn. Math. 44:2, 2019, 615-633.

[3] Brannan, D. A., W. H. J. Fuchs, W. K. Hayman, and Ü. Kuran: A characterization of harmonic polynomials in the plane. - Proc. London Math. Soc. (3) 32, 1976, 213-229.

[4] Davis, B.: Picard's theorem and brownian motion. - Trans. Amer. Math. Soc. 213, 1975, $353-362$.

[5] Eremenko, A., and J. Lewis: Uniform limits of certain A-harmonic functions with applications to quasiregular mappings. - Ann. Acad. Sci. Fenn. Ser. A I Math. 16, 1991, 361-375.

[6] Eremenko, A., and M. Sodin: Distribution of values of meromorphic functions and meromorphic curves from the standpoint of potential theory. - Algebra i Analiz 3:1, 1991, 131-164 (in Russian).

[7] Krantz, S.: Complex analysis: the geometric viewpoint. Second edition. - Carus Math. Monogr. 23, Math. Assoc. America, 2004.

[8] Kuran, Ü: Generalizations of a theorem on harmonic functions. - J. London Math. Soc. 41, 1966, 145-152. 
[9] Lewis, J.: Picard's theorem and Rickman's theorem by way of Harnack's inequality. - Proc. Amer. Math. Soc. 122:1, 1994, 199-206.

[10] Murdoch, B. H.: A theorem on harmonic functions. - J. London Math. Soc. 39, 1964, 581-588.

[11] Randsford, T.: Potential theory in the complex plane. - London Math. Soc. Stud. Texts, 1995.

[12] Remmert, R.: Classical topics in complex function theory. - Springer-Verlag, 1998.

[13] Rickman, S.: On the number of omitted values of entire quasiregular mappings. - J. Analyse Math. 37, 1980, 100-117.

[14] Saks, S., and A. Zygmund: Analytic functions. Third edition. - Elsevier, 1971.

[15] Schiff, J. L.: Normal families. - Universitext, Springer-Verlag, 1993.

Received 9 April 2019 • Accepted 11 October 2019 